\title{
Fault detection by surface seismic scanning tunneling macroscope: Field test
}

\author{
Sherif M. Hanafy* and Gerard T. Schuster, King Abdullah University of Science and Technology, Division of \\ Physical Science and Engineering, Thuwal 23955-6900. Saudi Arabia
}

\section{SUMMARY}

The seismic scanning tunneling macroscope (SSTM) is proposed for detecting the presence of near-surface impedance anomalies and faults. Results with synthetic data are consistent with theory in that scatterers closer to the surface provide brighter SSTM profiles than those that are deeper. The SSTM profiles show superresolution detection if the scatterers are in the near-field region of the recording line. The field data tests near Gulf of Aqaba, Haql, KSA clearly show the presence of the observable fault scarp, and identify the subsurface presence of the hidden faults indicated in the tomograms. Superresolution detection of the fault is achieved, even when the $35 \mathrm{~Hz}$ data are lowpass filtered to the $5-10 \mathrm{~Hz}$ band.

\section{INTRODUCTION}

Huang et al. (2014) presented the theory of the seismic scanning tunneling macroscope (SSTM) for surface reflection data. The objective is to detect the presence of near-surface impedance anomalies. The key idea in their paper is that the shot gathers can be used as natural Green's functions, so the reflections can be naturally migrated to the recording line without knowing the velocity model.

The presence of near-field scatterers can be detected by large amplitudes in the migration profile along the recording line. We shall denote this migration profile a seismic scanning tunneling macroscope (SSTM) profile (Schuster et al., 2012). There are two important features in the SSTM profile that could be used to detect near-surface faults.

1. If the subwavelength faulting is in the near field of the recording line then the scattered wavefield can be used to detect the fault location with superresolution accuracy. If the scattered energy is significantly less than the direct wave energy then the correlation of the direct with itself will mask the fault's presence.

2. The correlation of the direct wave with the scattered arrivals can be used to detect the presence of the fault in the near field of the recording line. Larger amplitudes in the SSTM migration profile suggest a greater density of impedance anomalies, such as a fault scarp.

In this current paper we test the ability of the SSTM profile to detect a fault for seismic data collected near the Gulf of Aqaba, Haql, KSA. The fault break is seen on the surface, so it is the ground-truth to assess the detection accuracy of this method. There are also buried faults revealed by a tomogram, so we will determine if the SSTM profile can detect these as well.

The next section briefly outlines the theory of the SSTM, then numerical results are presented. Elastic simulations are used to validate the capability of using the SSTM in detecting impedance anomalies with data recorded by a recording geometry similar to that of the Aqaba data. The SSTM is then tested on the Aqaba data. Finally, conclusions are presented.

\section{THEORY}

Assume the surface acquisition geometry in Figure 1, where the scatterer is in the near field of the point source at $\mathbf{s} \in \mathbf{B}$ so that the evanescent portion (denoted as a wiggly arrow) of the source field interacts with the subwavelength scatterer at $\mathbf{x}_{0}$, converts to a body wave, and propagates (denoted as a straight ray) to the receivers on the surface denoted by $\mathbf{B}$. For a single scatterer located at $\mathbf{x}_{o}$, we will denote the recorded scattered field in the frequency domain as

$$
\begin{aligned}
& \mathbf{g} \in B ; \mathbf{s} \in B \\
& d(\mathbf{g} \mid \mathbf{s})_{\text {sct }}=r_{o} G\left(\mathbf{g} \mid \mathbf{x}_{o}\right) G\left(\mathbf{x}_{o} \mid \mathbf{s}\right),
\end{aligned}
$$

where $d(\mathbf{g} \mid \mathbf{s})_{\text {sct }}$ will be denoted as the 0th-order scattering Green's function for a harmonic point source at $\mathbf{s}$ and geophone at $\mathbf{g}$, and $r_{o}$ is the scattering coefficient ${ }^{1}$. The free-space Green's function in a homogeneous medium is represented by $G(\mathbf{g} \mid \mathbf{s})$.

\section{Scattering from a Near -field Scatterer}

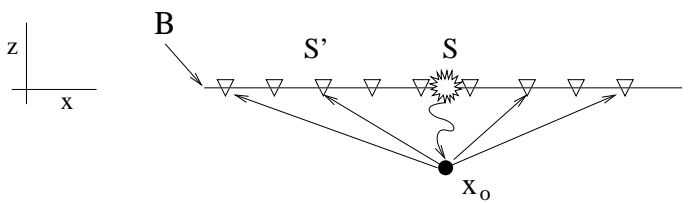

Figure 1: Single scatterer (black solid circle) in the near-field region of the source line. The point source location at $\mathbf{s}$ here is just next to the scatterer at $\mathbf{x}_{o}$.

To migrate the scattered arrivals back to the source line, the scattered data $d(\mathbf{g} \mid \mathbf{s})_{\text {sct }}$ are multiplied by the natural migration kernel $d\left(\mathbf{g} \mid \mathbf{s}^{\prime}\right)_{\text {sct }}^{*}$ and the product is summed over all geophone positions to get the prestack migration image $m\left(\mathbf{s}^{\prime}, \mathbf{s}\right)$ at the trial image point $\mathbf{s}^{\prime} \in B$ :

$$
\begin{gathered}
\\
m\left(\mathbf{s}^{\prime}, \mathbf{s}\right)=\sum_{g \in B} d(\mathbf{g} \mid \mathbf{s})_{\mathrm{sct}} d\left(\mathbf{g} \mid \mathbf{s}^{\prime}\right)_{\mathrm{sct}}^{*}, \\
=r_{o}^{2} G\left(\mathbf{x}_{o} \mid \mathbf{s}\right) G\left(\mathbf{x}_{o} \mid \mathbf{s}^{\prime}\right)^{*} \sum_{g \in B_{g}}\left|G\left(\mathbf{g} \mid \mathbf{x}_{o}\right)\right|^{2},
\end{gathered}
$$

for a point source at $\mathbf{s} \in B$ just next to the scatterer at $\mathbf{x}_{o}$. The migration image $m\left(\mathbf{s}^{\prime}, \mathbf{s}\right)$ is restricted to be along the source and

\footnotetext{
${ }^{1}$ To minimize notational clutter, we assume that the source wavelet is $W(\omega) \propto 1 / \omega^{2}$ to avoid the term $\omega^{2} / c^{2}$ seen in the Born modeling formula
} 


\section{Fault detection by SSTM: Field test}

receiver line $\mathbf{B}$ because we only record $G\left(\mathbf{x} \mid \mathbf{x}^{\prime}\right)$ for $\mathbf{x}, \mathbf{x}^{\prime} \in \mathbf{B}$, where its conjugate is the exact scattering migration kernel. Here, the stacked migration image $\tilde{m}\left(\mathbf{s}^{\prime}\right)$ for $\mathbf{s}^{\prime} \in B$ is given by,

$$
\tilde{m}\left(\mathbf{s}^{\prime}\right)=\sum_{s \in B} \sum_{g \in B} d(\mathbf{g} \mid \mathbf{s})_{\text {sct }} d\left(\mathbf{g} \mid \mathbf{s}^{\prime}\right)_{\text {sct }}^{*}
$$

and in practice we also sum over all frequencies; the summation of these prestack images gives the stacked migration profile. This migration image should indicate the location along $\mathbf{B}$ of near-surface scatterers with superresolution capability. For more details on the theory see Schuster et a. (2012) and Huang et al. (2014).

\section{NUMERICAL TESTS}

There will be four-synthetic and one-field data tests of the SSTM for detecting the presence of near-surface impedance anomalies.

\section{Synthetic Example}

The SSTM method is tested on synthetic data simulated for two velocity models. The first one is a homogenous model with a P-wave velocity of $1500 \mathrm{~m} / \mathrm{s}$, a S-wave velocity of 750 $\mathrm{m} / \mathrm{s}$, and a density of $2.0 \mathrm{gm} / \mathrm{cc}$ (Figure $2 \mathrm{a}$ ). While the second one is similar to model 1, except clusters of scatterers are incorporated into the model. Each cluster is a grid of $3 \times 3$ scatterer points, each point has an area of $6 \times 6 \mathrm{~m}^{2}(0.2 \lambda \times 0.2 \lambda$, where $\lambda$ is the wavelength) and the offset between each neighboring gridpoints is $6 m$ (Figure $2 \mathrm{f}$ ). The depths to the scatterer clusters are 4,10 , and $16 \mathrm{~m}(0.1,0.3$, and $0.4 \lambda)$. The scatterer has a P-wave velocity of $2000 \mathrm{~m} / \mathrm{s}$, a S-wave velocity of $1000 \mathrm{~m} / \mathrm{s}$, and a density of $2.3 \mathrm{gm} / \mathrm{cc}$. A total of 400 sources and receivers are located along the top surface with $2 \mathrm{~m}$ sampling intervals. The source wavelet is a Ricker wavelet with a $20-\mathrm{Hz}$ peak frequency. Shot gathers for both models are generated using a finite-difference solution to the $2 \mathrm{D}$ elastic wave equation.

\section{Model 1: Halo Test.}

All traces $d(\mathbf{g} \mid \mathbf{s})$ within an offset of one wavelength from the source location at $\mathbf{s}$ are muted to create the halo shot gathers. These near-offset traces are muted in order to mitigate the strong effects of the direct-direct correlation in equation 2 so that the direct-scattered correlations are emphasized in the migration profile. The halo shot gathers are then used to calculate the SSTM pre-stack profiles shown in Figure (2b). Since the velocity model is homogenous, the SSTM pre-stack profiles (2b) and the stacked SSTM curve (Figure 2c) do not vary too much except at the left and right sides of the curve, which is an expected truncation effect.

\section{Model 1: Mute Direct-Arrivals Test.}

The direct P- and S-wave arrivals are muted and the muted shot gathers are then used to calculate the SSTM profiles shown in Figure (2d). The SSTM pre-stack image show a smoothly varying profile and the stacked SSTM curve (Figure 2e) gradually increases toward the center. We can notice a fast decrease in values at the far-left and far-right sides of the curve, which is a truncation artifact.

\section{Model 2: Halo Test}

All traces within an offset of one wavelength from the source location are muted to create the halo shot gathers. The halo shot gathers are then used to calculate the SSTM curves shown in Figure (2g) and the effects of the three scatterer clusters (A, $\mathrm{B}$, and C) are shown on the SSTM pre-stack image. The effect of cluster $\mathrm{A}$ is greater than that for $\mathrm{B}$, which is larger than that for $\mathrm{C}$. This is due to the differences in the depth to the scatterer clusters (Figure 2f). The closer the scatterer points to the source line the stronger its effect on the SSTM pre-stack image. Although the effect of the scatterer clusters is shown on the pre-stacked SSTM image, it does not appear in the stacked profile (Figure $2 \mathrm{~h}$ ), and this is likely due to the direct arrivals in the shot gathers. Strong direct arrivals will tend to smooth the SSTM profiles.

\section{Model 2: Mute Direct-Arrivals Test.}

To avoid the effect of the strong direct arrivals we muted the direct P- and S-wave arrivals, where only scatterer effects is kept in the shot gathers. Then the muted shot gathers are used to calculate the SSTM curves shown in Figure (2i). The SSTM pre-stack image show high values at the center, which is due to the scattered energy from the scatterer clusters. The stacked SSTM curve (Figure 2j) shows high values corresponding to the first scatterer cluster (A) compared to the weaker energy from the second and the third scatterer clusters (B and C).

We can summarize the observations from the synthetic examples:

- To differentiate between peaks due to scatterers and peaks due to truncation effect at the stacked SSTM we need to look at both the pre-stack and the stacked SSTM profiles. Peaks at the side of the stacked profile and not seen on the pre-stack profiles are mainly due to truncation artifacts.

- The effect of the scatterer point on the SSTM image is inversely proportional to the distance between the scatterer point and the source line; if this offset is large $(>0.5 \lambda)$, then it will be difficult to see its effect on the SSTM image.

\section{Field Data Example}

A data set recorded along the north western part of the Saudi coast of the Gulf of Aqaba is used to test the proposed SSTM method. This data set will be used to locate a surface fault that ruptured in 1995 (Figure 3). The data set contains 120 shot gathers with 120 trace/shot gather (Figure 5a), where the source/receiver offset is $2.5 \mathrm{~m}$ (Figure 4). The first-arrival traveltimes are picked and then inverted to generate the traveltime tomogram shown in Figure 5c.

The recorded seismic data are used to test the SSTM. The traces within an offset of one wavelength from the shot location (halo test) and the early arrival events are muted, which are then used to generate the SSTM pre-stack profiles shown in 


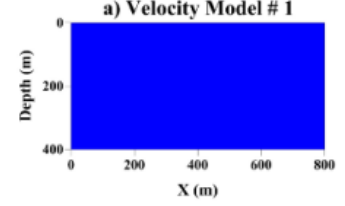
Hale Test

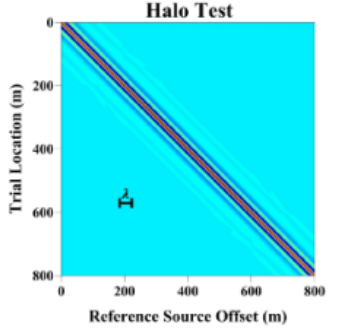

c) SSTM Stack Profile Halo Test

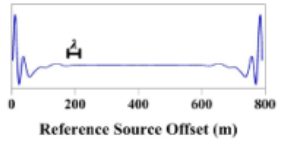

d) SSTM Pre-stack Profiles

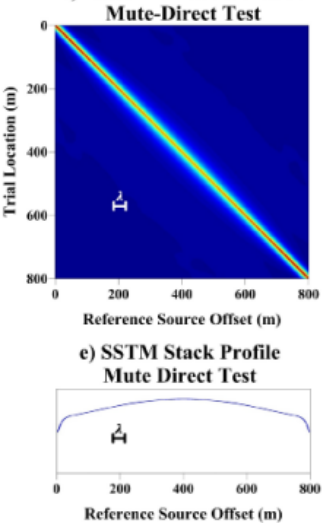

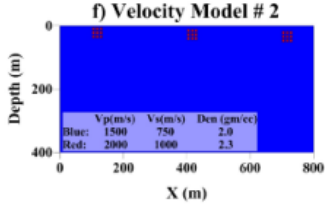

g) SSTM Pre-stack Profiles Halo Test

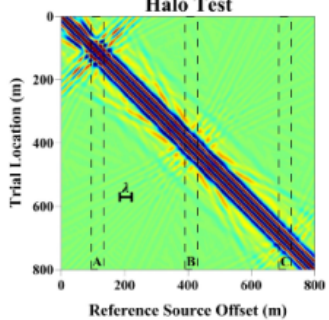

h) SSTM Stack Profile Halo Test

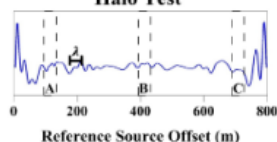

i) SSTM Pre-stack Pron

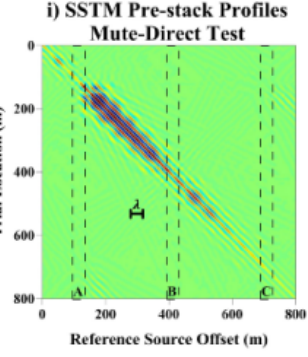

j) SSTM Stack Profil Mute Direct Test

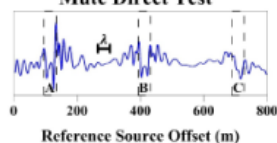

Figure 2: a) Velocity model 1 . b) The pre-stack 2D SSTM image of model 1 using the halo data, the halo size is one wavelength at each side of the source location. The black line represents the wavelength $\lambda$. c) The stacked SSTM curve for velocity model 1 with halo data set. d) The pre-stack 2D SSTM image of model 1 where, we muted the early arrivals from the shot gathers. e) The stacked SSTM curve for the velocity model with muted-early-arrivals data set. f) Velocity model 2. g) The pre-stack 2D SSTM image of model 2 using halo data, here, the halo size is one wavelength at each side of the source location. h) The stacked SSTM curve for velocity model 2 with the halo data. i) The pre-stack 2D SSTM profile of model 2 , here, we muted the early arrivals from the shot gathers. j) The stacked SSTM profile for velocity model 2 with muted-early-arrivals data.

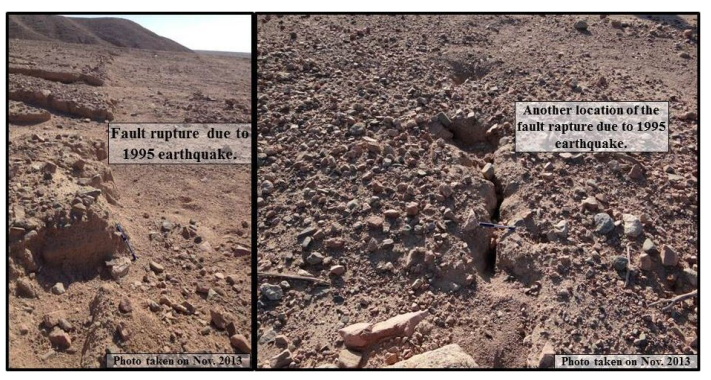

Figure 3: Two photos show the rupture due to the 1995 earthquake.

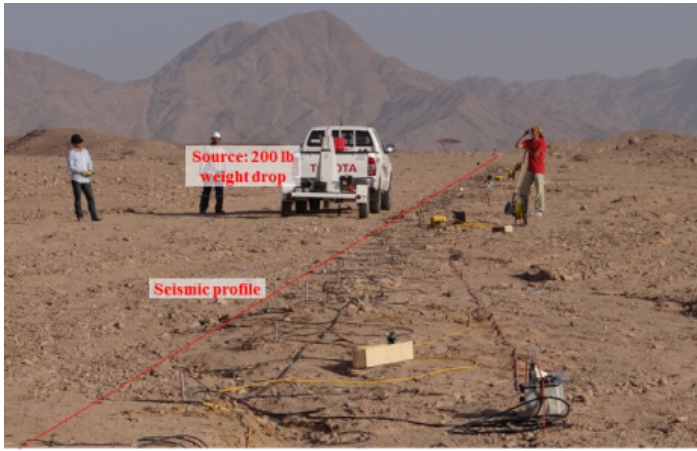

Figure 4: Acquisition of seismic data at the Gulf of Aqaba, north western of Saudi Arabia.
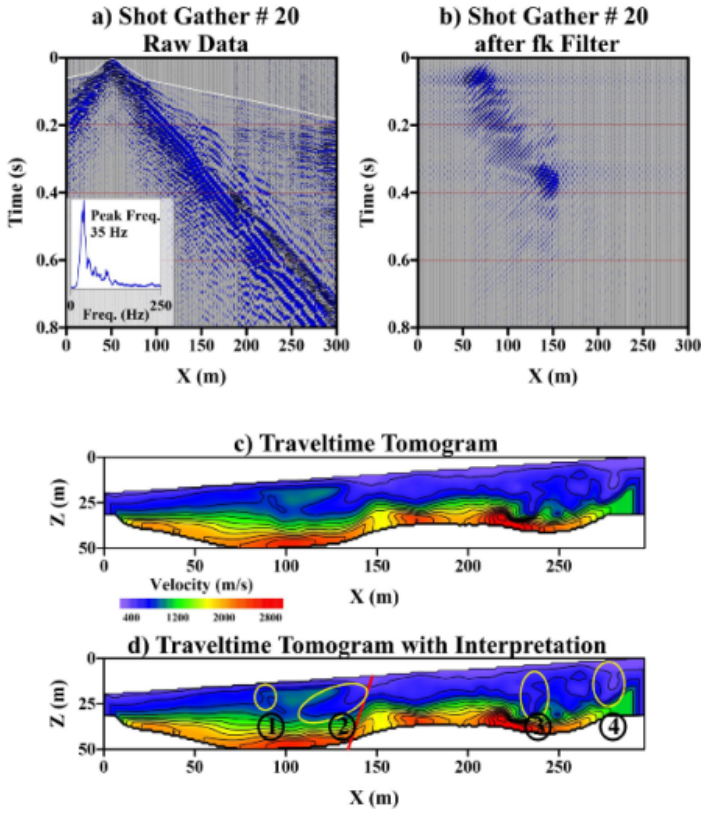

Figure 5: a) Common shot gather no. 20. The data characterized by high signal-to-noise ratio, which increased the accuracy of first-arrival traveltime picking. White line shows the first arrival event. b) Same as a) after fk filter to keep only back-scattering events from the fault. c) The traveltime tomogram generated by inverting the first-arrival traveltimes. d) The traveltime tomogram with interpretation. The rupture due to the 1995 earthquake is shown at an offset $\mathrm{X}=133-148 \mathrm{~m}$. 


\section{Fault detection by SSTM: Field test}

Figure (6a) and its corresponding stacked SSTM profile shown in Figure (6b). High amplitude values appear as three peaks in the stacked profile between offsets 133 and $148 \mathrm{~m}$, the effect of its corresponding colluvial wedge is shown between offsets 108 and $145 \mathrm{~m}$. Another anomaly appears between offsets 270 and $285 \mathrm{~m}$, which could be due to the low-velocity anomaly seen in the traveltime tomogram.

A fk filter is applied to the data to remove all events except the back-scattering due to the fault. The filtered data is then used to generate the SSTM pre-stack (Figures 6c) and stacked (Figures 6d) images. The fault effect is shown as large amplitudes in the pre-stack and stacked SSTM profiles. The amplitude value on the SSTM profiles due to the low-velocity anomaly located at the offset 270-285 m increased. The two anomalies located at offsets 83-94 $\mathrm{m}$ and 230-240 $\mathrm{m}$ in the tomogram do not produce large amplitudes in the SSTM stacked profile, while the anomaly at offset $230-240 \mathrm{~m}$ is shown only on the pre-stack SSTM image. This indicates that the anomaly located at offset 83-94 $\mathrm{m}$ is too deep or too small to be detected in the SSTM profile, while the anomaly at offset $230-240$ is at an intermediate depth so that it is detected on the pre-stack image. However, it has a very weak effect on the stacked profile (similar to the scatterer cluster B in the synthetic example).
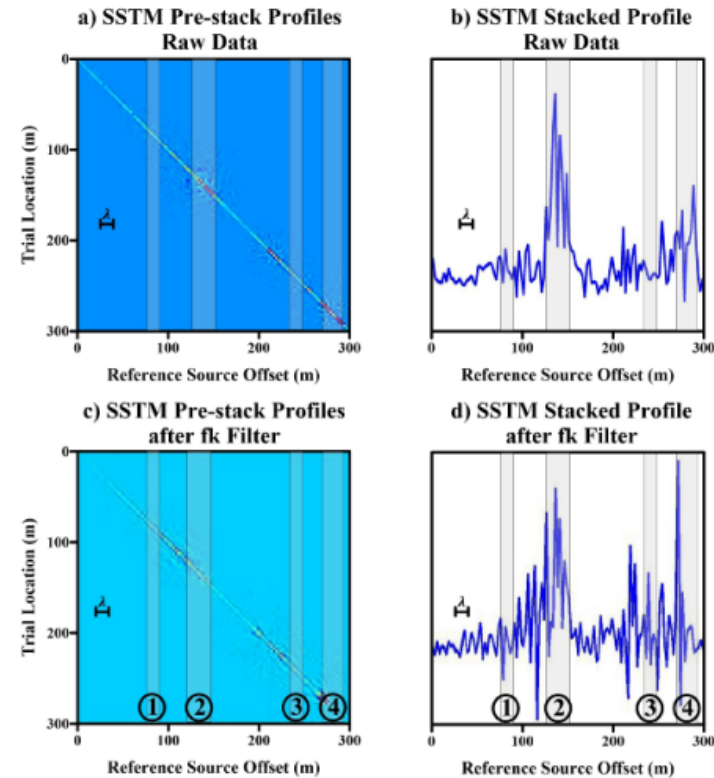

d) SSTM Stacked Profile after fk Filter

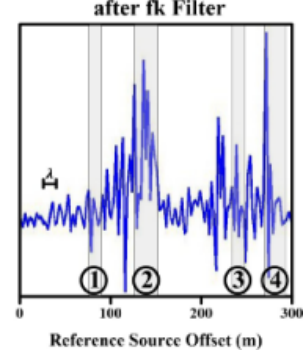

Figure 6: a) and b) The SSTM pre-stack image and stacked profile, respectively of the raw data. c) and d) same as a) and b) except after apply band-pass filter 5-10 Hz. Note in d) that the wavelength increased since the peak frequency decreased after band-pass filter. e) and f) as as a) and b) except after apply band-pass filter 5-100 Hz.

\section{CONCLUSIONS}

The SSTM method is used to detect the presence of near-surface impedance anomalies and faults. Results with synthetic data are consistent with theory in that scatterers closer to the sur- face provide brighter SSTM profiles than those that are deeper. They also show superresolution capability if the scatterers are in the near-field region of the recording line. The field data tests clearly show the presence of the observable fault scarp, and detect the presence of the hidden faults indicated in the tomograms. The next step is to extrapolate the recorded Green's functions to different depths and use them to obtain migration profiles in depth, not just offset.

\section{ACKNOWLEDGEMENT}

We would like to thank the 2013 sponsors of the CSIM Consortium for their support. We would like to thank KAUST and the hpc department (SHaheen) to the supportthey providedto complete this work. 
http://dx.doi.org/10.1190/segam2014-1013.1

\section{EDITED REFERENCES}

Note: This reference list is a copy-edited version of the reference list submitted by the author. Reference lists for the 2014 SEG Technical Program Expanded Abstracts have been copy edited so that references provided with the online metadata for each paper will achieve a high degree of linking to cited sources that appear on the Web.

\section{REFERENCES}

Huang, Y., G. T. Schuster, and Sh. M. Hanafy, 2014, Fault detection by SSTM: Presented at the CSIM Annual Meeting.

Schuster, G. T., Sh. M. Hanafy, and Y. Huang, 2012, Theory and feasibility tests for a seismic scanning tunnelling macroscope: Geophysical Journal International, 190, no. 3, 1593-1606, http://dx.doi.org/10.1111/j.1365-246X.2012.05564.x. 